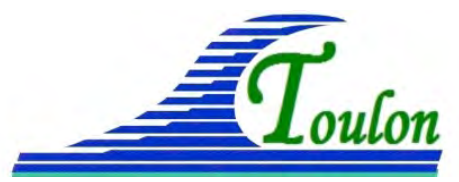

XIV èmes Journées Nationales Génie Côtier - Génie Civil Toulon, 29 juin au $1^{\text {er }}$ juillet 2016

DOI:10.5150/jngcgc.2016.023 (C) Editions Paralia CFL

disponible en ligne - http://www.paralia.fr - available online

\title{
Influence des conditions hydrométéorologiques sur les flux et bilans sédimentaires en estuaire de Seine
}

\section{Florent GRASSO ${ }^{1}$, Elisabeth SCHULZ ${ }^{1}$, Pierre LE HIR ${ }^{1}$, Romaric VERNEY ${ }^{1}$, Bénédicte THOUVENIN ${ }^{1}$}

\section{IFREMER, DYNECO-DHYSED, BP 70, 29280 Plouzané, France.}

florent.grasso@ifremer.fr

\section{Résumé :}

La dynamique sédimentaire à l'embouchure de l'estuaire de Seine est principalement contrôlée par les forçages hydrométéorologiques (débit, vent, vagues, marée) et les fortes pressions anthropiques (endiguement, dragage, clapage). La compréhension et la quantification de la dynamique des flux sédimentaires en suspension (sable/vase) est un enjeu majeur, autant d'un point vu écologique (ex. turbidité, envasement) que financier (ex. gestion des chenaux de navigation). La mise en place de réseaux de mesure in situ permet une meilleure compréhension de la dynamique sédimentaire de l'estuaire, mais leur caractère local rend difficile toute estimation des flux et bilans sédimentaires. Pour répondre à ces questions, cette étude s’appuie sur la modélisation numérique, validée par de nombreuses séries de mesures in situ, afin de quantifier les flux et bilans sédimentaires à différentes échelles caractéristiques de temps et d'espace.

Les concentrations simulées dans la zone d'extension du bouchon vaseux sont du même ordre que les observations et leur dynamique associée aux différentes phases et cycles de la marée, ainsi qu'au cycle hydrologique, est satisfaisante. L'analyse des flux résiduels et bilans sédimentaires aux échelles annuelle et trimestrielle a permis d'identifier un schéma de circulation à l'embouchure de l'estuaire et de quantifier l’importance du forçage hydrologique (crue/étiage) sur les échanges sédimentaires. Enfin, ce travail se poursuivra par l'étude de ces flux et bilans pour différents scénarios hydrométéorologiques contrastés.

Mots-clés : Dynamique hydro-sédimentaire, sable/vase, Estuaire de Seine, Modélisation numérique, Mesures in situ, Dragage, Flux résiduels et bilans, Année/trimestres.

\section{Introduction}

Les flux sédimentaires de l'estuaire de Seine dépendent fortement de la dynamique du bouchon vaseux (BRENON et al., 1999; GRASSO et al., 2014), comme cela est observé pour de nombreux estuaires (ex. YU et al., 2014 ; MCSWEENEY et al., 2016 ; JALON-ROJAS et al., 2016; TOUBLANC et al., 2016). De plus, la remise en suspension des sédiments sur les bancs à l'embouchure et les opérations de dragage le long des chenaux de navigation jouent un rôle très important (AVOINE et al., 1981 ; LE HIR et al., 2001 ; MARMIN et al., 2014). Dans cet estuaire macrotidal, la dynamique 


\section{Thème 2 - Dynamique sédimentaire}

sédimentaire est contrôlée par les forçages hydrométéorologiques agissant à différentes échelles temporelles : variations de débit (mois), cycles de marée vive eau/morte eau (VE/ME) (semaines), tempêtes (jours), phases de marée (heures). L'estimation des flux et bilans sédimentaires dans le système intra-estuarien et à ses limites amont/aval, ainsi que leur variabilité face aux conditions hydrométéorologiques, représente une forte attente de la part de la communauté scientifique et des gestionnaires. Cela nécessite une connaissance de la vitesse des courants et des concentrations en matières en suspension (MES) le long de nombreuses sections de l'estuaire, ce qui est accessible grâce aux modèles numériques hydro-sédimentaires tridimensionnels (ex. LE HIR, 2009). Il existe des mesures in situ de MES réalisées le long de l'estuaire, en particulier avec la mise en place du réseau de mesure SYNAPSES (GIPSA, page web), permettant une meilleure compréhension de la dynamique sédimentaire de l'estuaire. Cependant, leur caractère local rend difficile toute interprétation sur leur représentativité spatiale et ne permet pas de quantifier directement les flux et bilans sédimentaires. L'objectif de cette étude est donc d'analyser par modélisation tridimensionnelle la dynamique des sédiments en suspension dans l'estuaire de Seine, afin de quantifier les flux et bilans sédimentaires à différentes échelles caractéristiques de temps et d'espace. Ce travail vient compléter les rares simulations de flux sédimentaires en estuaire de Seine (ex. LE HIR, 2009), avec un modèle hydro-sédimentaire plus avancé, permettant une meilleure description des processus physiques, et une validation de la dynamique sédimentaire plus fine.

\section{Matériel et méthodes}

\subsection{Modélisation numérique}

Ce travail se base sur le modèle hydrodynamique MARS3D (LAZURE \& DUMAS, 2008) de l'estuaire à la baie de Seine (figure 1) forcé aux limites par les composantes principales de la marée, le vent issu du modèle Météo France ARPEGE et le débit réaliste de la Seine mesuré au barrage de Poses en limite amont du modèle (point $\mathrm{P}$ sur la figure 1a). Les vagues sont simulées à l'aide du modèle WAVE WATCH III ${ }^{\circledR}$ (ARDHUIN \& ROLAND, 2012), depuis l'océan Atlantique vers un modèle local de même résolution que le modèle de circulation. Le modèle hydrodynamique est couplé au modèle sédimentaire multi-couches, multi-classes (sable/vase) SEDIMARS (LE HIR et al., 2011), prenant en compte les processus d'érosion, suspension, dépôt et consolidation (GRASSO et al., 2015). Cinq classes de sédiment sont initialement distribuées selon les cartes de faciès sédimentaires de l'estuaire de Seine (LESOURD $e t$ al., In press) : un gravier (diamètre médian $d_{50}=5 \mathrm{~mm}$ ), trois sables $\left(d_{50}=800 \mu \mathrm{m}\right.$, $\left.d_{50}=210 \mu \mathrm{m}, d_{50}=100 \mu \mathrm{m}\right)$ et une vase $\left(d_{50}=20 \mu \mathrm{m}\right)$ de vitesse de chute variable $w_{s}=[0,1-1,5 \mathrm{~mm} / \mathrm{s}]$ (VAN LEUSSEN, 1988). Cependant, les classes de sédiments grossiers ne prennent pas en compte la présence de débris coquilliers, comme cela peut être observé en nature. 


\section{XIV èmes Journées Nationales Génie Côtier - Génie Civil \\ Toulon, 29 juin au $1^{\text {er }}$ juillet 2016}

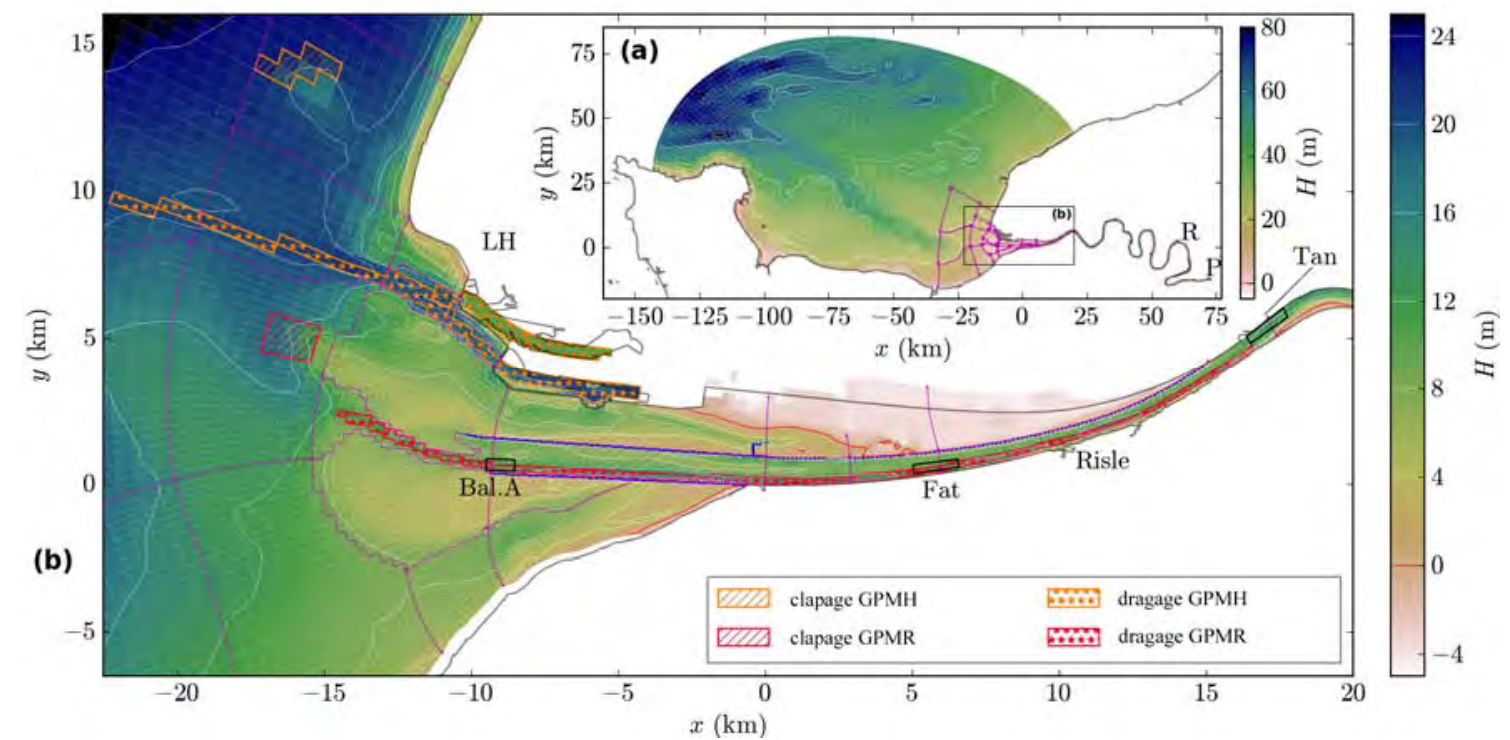

Figure 1. Bathymétrie du domaine complet (a) couvert par le modèle et zoom (b) sur l'embouchure de l'estuaire de Seine. Les lignes violettes délimitent les zones de calcul des flux et bilans sédimentaires, les zones étoilées (resp. hachurées) représentent les zones de dragage (resp. de clapage) du GPMH (orange) et du GMPR (rouge), les cadres noirs à Balise A (Bal.A), Fatouville (Fat) et Tancarville (Tan) représentent les zones de comparaison des MES mesurées/simulées.

Le modèle prend en compte les zones draguées par les Grands Ports Maritimes du Havre (GPMH) et de Rouen (GPMR) (figure 1) en enlevant les sédiments lorsque que la cote de fond dépasse une cote de dragage et les redéposant dans la couche de fond de la colonne d'eau dans les zones de clapage.

Enfin, un maillage curviligne non-orthogonal est adopté afin de mieux respecter la forme de l'estuaire, d'optimiser les coûts de calcul avec des mailles de taille variable et d'améliorer l'estimation des flux sédimentaires. La dimension des mailles varie d'environ 50 m à l'embouchure jusqu'à 2 km au large.

\subsection{Mesures in situ}

Le travail de calibration et de validation des simulations numériques s'est appuyé sur les jeux de données très riches collectés dans le cadre des programmes de recherche SeineAval et du réseau de mesure SYNAPSES (élévation de la surface libre, courant, salinité et turbidité). Les données in situ présentées dans cette étude concernent les mesures de turbidité 2014-2015 issues du réseau SYNAPSES aux points Balise A (bouée), Fatouville (ponton) et Tancarville (ponton) (figure 1) qui encadrent l'étendue du bouchon vaseux au cours de son cycle hydrologique annuel. Le débit moyen sur l'année août 2014 - août 2015 est de $516 \mathrm{~m}^{3} / \mathrm{s}$, représentatif du débit moyen des vingt dernières années $\left(\sim 500 \mathrm{~m}^{3} / \mathrm{s}\right)$. Des études réalisées dans le cadre du projet FLUMES (Seine- 


\section{Thème 2 - Dynamique sédimentaire}

Aval 4 ; VERNEY, 2012) mettent en évidence une forte variabilité du coefficient de calibration entre la turbidité mesurées en NTU ( $\left.t u r b_{N T U}\right)$ et la concentration en g/l des matières en suspension $\left(C_{M E S}\right)$ vis-à-vis des variations hydrologiques et tidales. Il a donc été décidé d'utiliser un coefficient de calibration $\left(c_{c a l}\right)$ moyen associé à une incertitude tel que $C_{M E S}(\mathrm{~g} / \mathrm{l})=c_{c a l} \times \operatorname{turb} b_{N T U}$, avec $c_{c a l}=0,0015 \pm 0,0005$. De plus, des mesures complémentaires, issues de campagnes en mer réalisées dans les chenaux en face des pontons de Fatouville et Tancarville, peuvent faire apparaitre des variations significatives en termes de phasage et d'intensité, nous amenant ainsi à relativiser les valeurs des mesures in situ. Afin de réduire l'incertitude sur les mesures de turbidité, il serait intéressant dans le futur de développer une relation MES-NTU variable en fonction du cycle hydrologique.

\section{Résultats}

\subsection{Validation et analyse de la dynamique des matières en suspension}

Suite à la validation du modèle hydrodynamique (KERVELLA et al., 2012), d'importants efforts ont été portés à la calibration et la validation des concentrations en MES afin de pouvoir quantifier de manière satisfaisante les flux et bilans sédimentaires à l'embouchure de l'estuaire de Seine. Les résultats de modèle présentés dans cette étude correspondent à des simulations réalisées après une année de spin up, afin de garantir la bonne tenue du bouchon vaseux après un cycle hydrologique complet (crue/étiage). Les comparaisons modèle/mesures se sont principalement basées sur les mesures de turbidité du réseau SYNAPSES entre août 2014 et août 2015 (Section 2.2). La figure 2 illustre les caractéristiques hydro-sédimentaires simulées par le modèle à Balise A, Fatouville et Tancarville. Les quantités tracées représentent les valeurs moyennes par zone d'étude (4x4 mailles, soit environ $400 \mathrm{~m}$ x $200 \mathrm{~m}$, voir figure 1), afin d'améliorer la représentativité des simulations. La hauteur d'eau moyenne aux différentes stations varie d'environ 10 à $12 \mathrm{~m}$ (figure 2a) et la contrainte de frottement au fond est essentiellement due aux courants de marée, excepté à Balise A en période de tempêtes (figure 2b). Les figures 2c, 2d et 2e représentent les concentrations médianes (percentile 50) en MES au cours d'un cycle de marée, mesurées et simulées. Le modèle simule relativement bien les MES mesurées aux stations, autant en termes d'intensité que de phasage tidal (VE/ME), avec toutefois une sous-estimation de l'intensité à Fatouville. Le phasage hydrologique est également bien simulé, avec une augmentation des MES à Balise $\mathrm{A}$ et diminution à Tancarville lorsque que le débit augmente (bouchon vaseux translaté à l'aval), et inversement lorsque le débit diminue. Cependant par fort étiage (ex. juillet 2015), les MES simulées à Tancarville sont sous-estimées, signifiant que le bouchon vaseux simulé semblerait ne pas être suffisamment en amont à cette période. 


\section{XIV vèmes Journées Nationales Génie Côtier - Génie Civil \\ Toulon, 29 juin au $1^{\text {er }}$ juillet 2016}
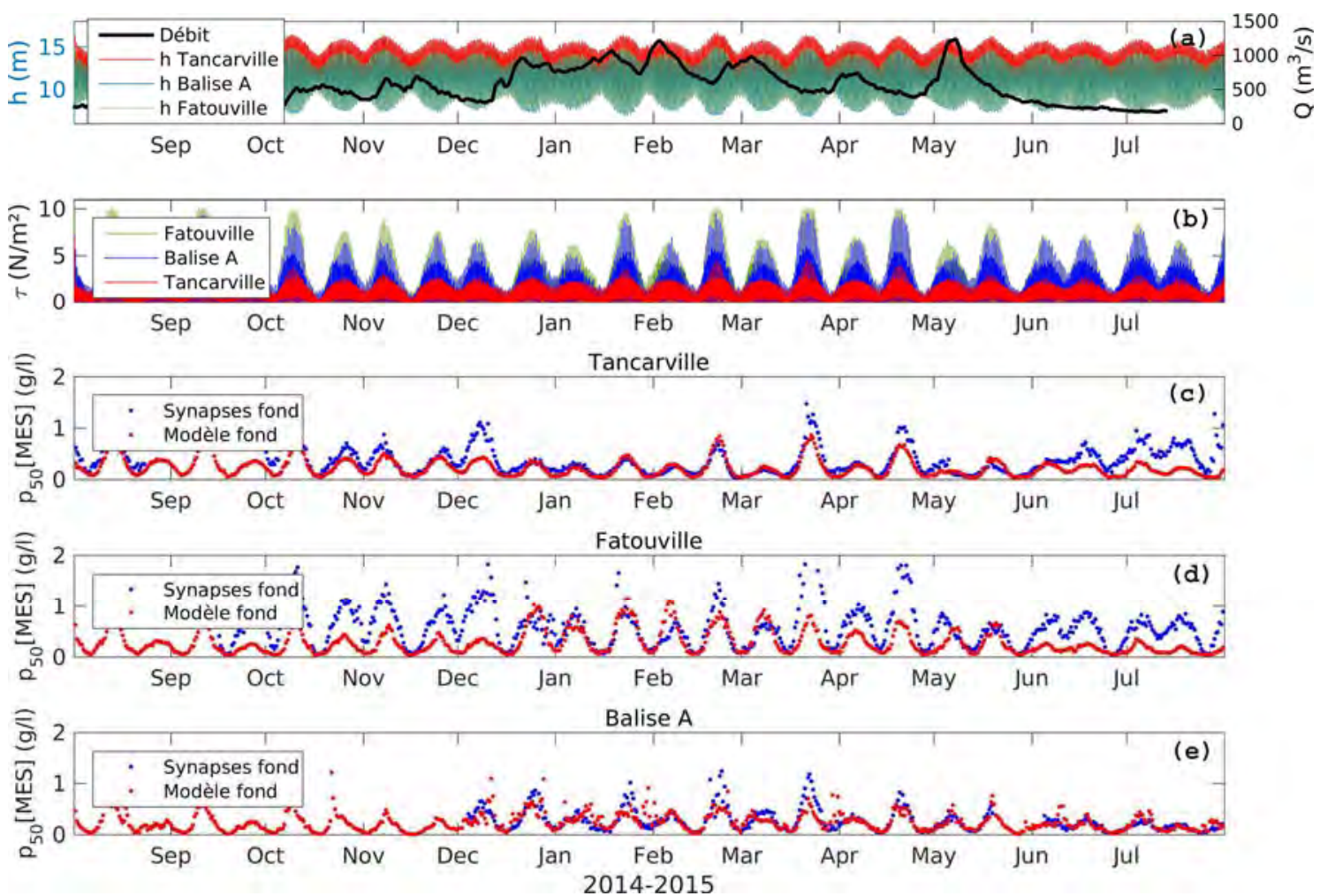

Figure 2. Evolution temporelle des caractéristiques hydro-sédimentaires d'août 2014 à août 2015. (a) Débit $Q$, hauteurs d'eau h et (b) contraintes de frottement au fond $\tau$ simulées à Balise A (bleu), Fatouville (vert) et Tancarville (rouge). Concentrations de MES médianes par cycle de marée mesurées par le réseau SYNAPSES (bleu) et simulées (rouge) à (c) Tancarville, (d) Fatouville et (e) Balise A.

De manière analogue à la figure 2, la figure 3 représente les caractéristiques hydrosédimentaires en période de crue (janvier 2015 à gauche) et par débit moyen (avril 2015 à droite), mais pour les MES mesurées et simulées toutes les 15 minutes. Les zones colorées représentent l'incertitude sur les mesures et la variabilité $\mathrm{min} / \mathrm{max}$ des simulations dans les cadres définis en Section 3.1 et illustrés figure 1. Les résultats mettent en évidence une forte variabilité des simulations entre les points proches des berges et ceux du chenal. La sous-estimation des MES à Fatouville est principalement marquée par débit moyen (figure 3i) mais le phasage tidal est dans l'ensemble correctement simulé aux différentes stations. Toutefois, les pics de concentration mesurés à Balise A aux étales de basse mer, probablement dus à la décantation du bouchon vaseux, sont généralement sous-estimés par le modèle (figures 3e et 3j).

Sachant que les masses sédimentaires draguées par les ports dans les chenaux de navigation menant au GPMH et GMPR représentent une part importante des flux sédimentaires à l'embouchure, il était nécessaire de confronter les simulations de dragage aux observations. De manière encourageante, les masses draguées dans le modèle sont proches des quantités annoncées par les ports (résultats non présentés ici). 

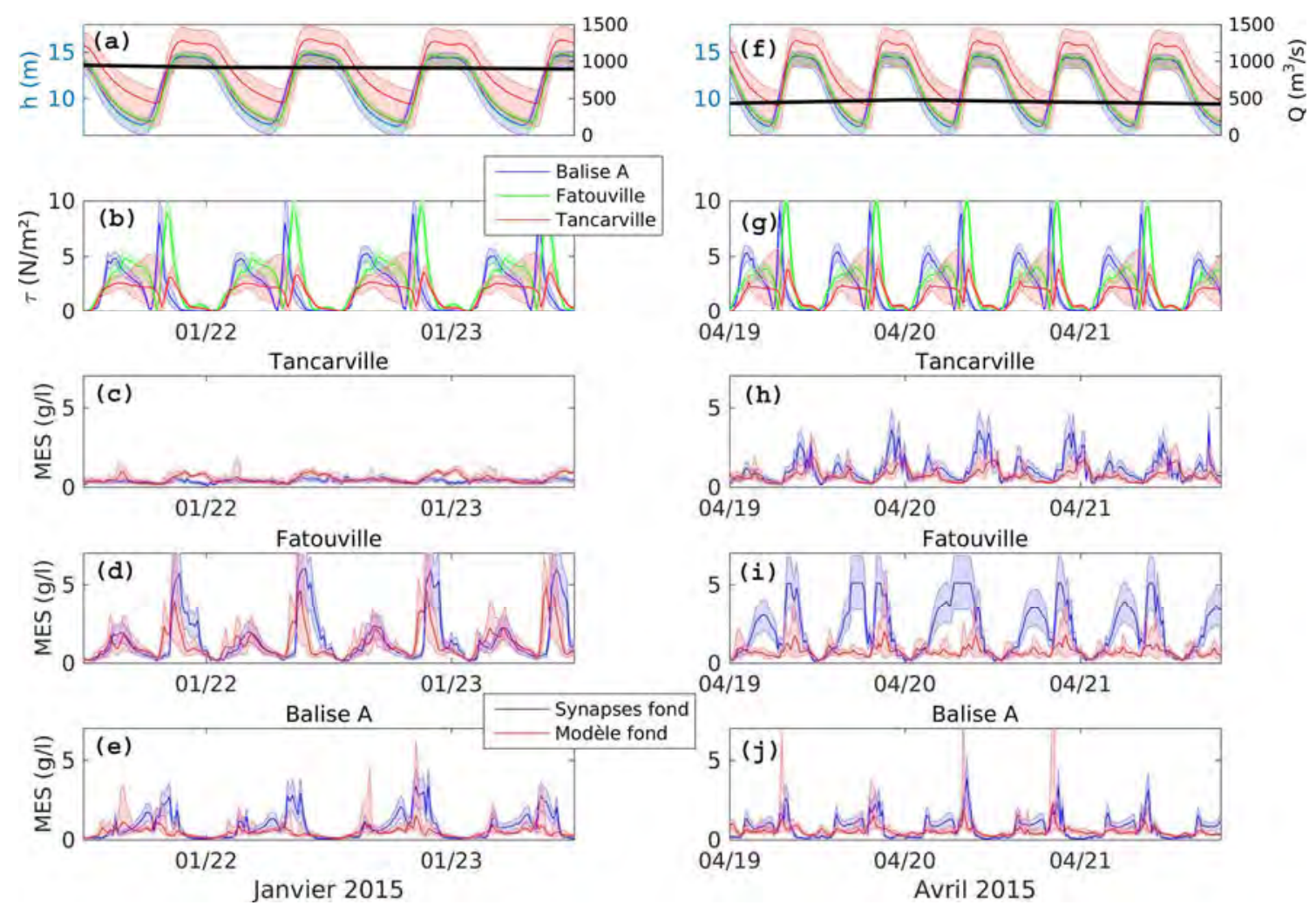

Figure 3. Evolution temporelle des caractéristiques hydro-sédimentaires (gauche) du 21 au 23 janvier 2015 et (droite) du 19 au 21 avril 2015. (a, f) Débit Q, hauteurs d'eau $h$ et $(b, g)$ contraintes de frottement au fond $\tau$ simulées à Balise A (bleu), Fatouville (vert) et Tancarville (rouge). Concentrations de MES moyennes mesurées par le réseau Synapses (bleu) et simulées (rouge) à $(c, h)$ Tancarville, $(d, i)$ Fatouville et $(e, j)$ Balise

A. Pour les simulations, les zones colorées représentent les variabilités min/max observées dans les cadres définis figure 1. Pour les mesures Synapses, les zones colorées représentent l'incertitude sur le coefficient de calibration des MES.

Par exemple au cours de l'année 2010-2011, le modèle simule 3,4 MT/an pour les zones GPMR ( 3,8 MT/an mesurées) et 2 MT/an pour les zones GPMH ( 1,6 MT/an mesurées). Le type de sédiment dragué par le modèle est principalement vaseux ( $65 \%$ de vase) pour les zones GPMH, en accord avec les observations ( $85 \%$ de vase) ; toutefois, il est sablo-vaseux ( $\sim 13 \%$ de vase et $\sim 64 \%$ de sable fin) pour les zones GPMR, alors que les observations soulignent davantage une vase sablonneuse ( $\sim 66 \%$ de vase et $\sim 28 \%$ de sable fin).

\subsection{Quantification des flux et bilans sédimentaires}

La quantification des flux et bilans sédimentaires s'est portée sur la période août 2010 août 2011, dont le cycle hydrologique (figure 4a) est également représentatif des 20 dernières années $\left(Q_{\text {moyen }}=426 \mathrm{~m}^{3} / \mathrm{s}\right)$. Plus de 25 zones réparties autour de l'embouchure 


\section{XIV ${ }^{e ̀ m e s}$ Journées Nationales Génie Côtier - Génie Civil \\ Toulon, 29 juin au $1^{\text {er }}$ juillet 2016}

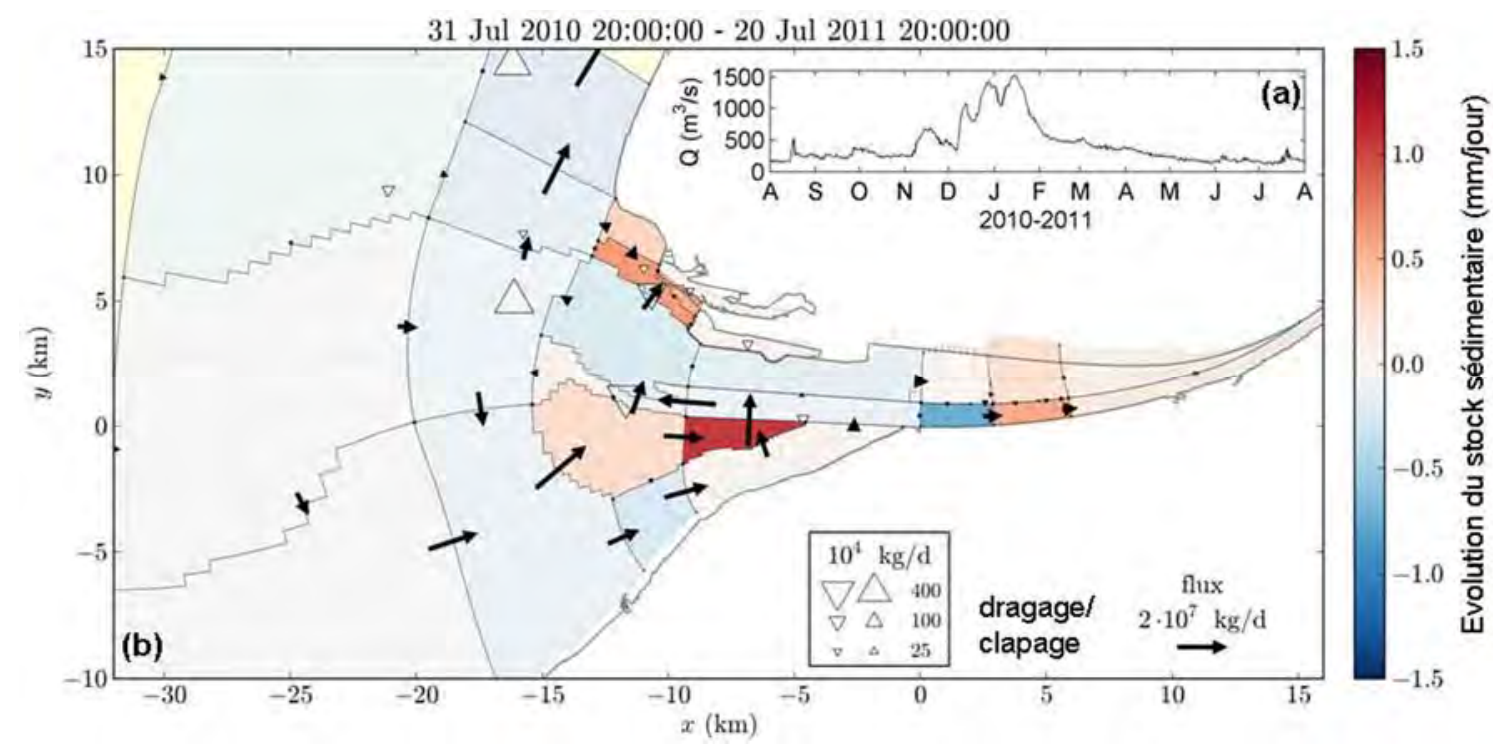

Figure 4. (a) Débit de la Seine et (b) flux résiduels (flèches), bilans sédimentaires (gain en rouge et perte en bleu), masses sédimentaires draguées (triangles vers le bas) et clapées (triangles vers le haut) entre le 31 juillet 2010 et le 20 juillet 2011.

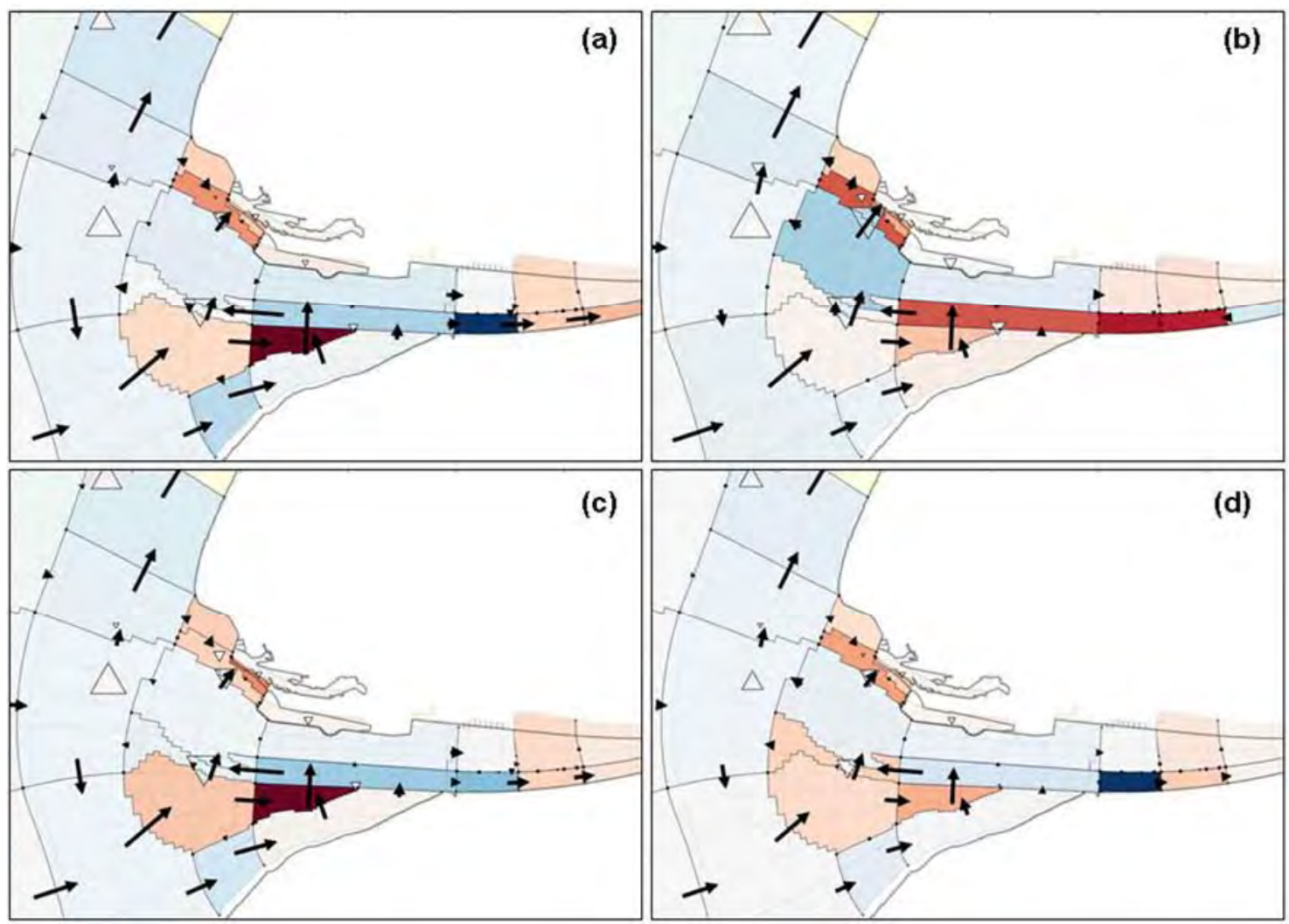

Figure 5. Même légende et échelle que la figure $4 b$ pour les périodes : (a) du 31 juillet au 28 octobre 2010, (b) du 28 octobre 2010 au 24 janvier 2011, (c) du 24 janvier au 23 avril 2011, et (d) du 23 avril au 20 juillet 2011. 


\section{Thème 2 - Dynamique sédimentaire}

de l'estuaire (figure 1) ont été définies afin de quantifier les échanges sédimentaires (gravier, sables et vase) entre les différentes sections caractéristiques de l'estuaire aval. La délimitation des zones s'est principalement basée sur les structures morphologiques de l'estuaire (ex. bancs/fosses à l'embouchure, chenaux principaux, vasières latérales, etc.) et sur les compartiments clés associés à la dynamique du bouchon vaseux. La figure 4b synthétise les échanges sédimentaires à l'échelle annuelle, à travers les flux résiduels (flèches) et les bilans sédimentaires (exprimés en équivalent $\mathrm{mm} / \mathrm{jour}$ d'accrétion ou d'érosion), ainsi que la contribution des dragages et clapages à ces bilans. Le schéma de circulation met en évidence une accrétion du banc sud-ouest à l'embouchure, ainsi qu'à l'aval de Fatouville, principalement due aux flux résiduels sableux. En revanche, les évolutions sédimentaires observées sur le reste de l'embouchure résultent majoritairement des flux de vase avec un dépôt notoire à l'ouest du GPMH entre le banc nord et le Cap de la Hève. De plus, il apparait nettement que les sédiments (principalement vaseux) sortant de l'estuaire, par advection naturelle et clapages, sont soit transportés vers le nord, soit réinjectés dans l'estuaire au sud-ouest.

La figure 5 illustre ces flux et bilans sédimentaire décomposés en quatre périodes d'environ trois mois. La période de crue (novembre-janvier, figure 5b) se différencie du reste de l'année avec un fort dépôt sédimentaire entre Balise A et Fatouville, principalement dû à la présence du bouchon vaseux dans cette zone à cette période, et un flux résiduel à Fatouville dirigé vers l'aval. Le dépôt à l'ouest du GPMH est également plus marqué et l'ensemble des quantités draguées est plus important. Même si le schéma de circulation antihoraire à l'embouchure est observé toute l'année, c'est durant les périodes août-octobre (figure 5a), février-avril (figure 5c) et mai-juillet 2010 (figure 5d) que les dépôts sur le banc sud-ouest sont les plus forts. Enfin, la période mai-juillet est nettement moins dynamique, autant en termes de flux et bilans sédimentaires, que de masses draguées.

\section{Conclusions}

Une modélisation hydro-sédimentaire sable/vase tridimensionnelle a été mise en place en estuaire de Seine afin d'étudier la dynamique des sédiments en suspension et quantifier les flux et bilans sédimentaires à différentes échelles spatiales et temporelles. Un important travail de calibration et validation des concentrations en matières en suspension a été mené, en s'appuyant sur de nombreuses mesures de turbidités collectées en estuaire de Seine. Les concentrations simulées dans la zone d'extension du bouchon vaseux sont du même ordre que les observations et leur dynamique associée aux différentes phases et cycles de la marée, ainsi qu'au cycle hydrologique, est satisfaisante.

La quantification des flux résiduels et bilans sédimentaires s'est portée sur différentes zones clés de l'estuaire aval au cours de la période août 2010 - août 2011. A l'échelle annuelle, un schéma de circulation à l'embouchure de l'estuaire a été identifié, associé à 


\section{XIV ${ }^{\text {èmes }}$ Journées Nationales Génie Côtier - Génie Civil \\ Toulon, 29 juin au $1^{\text {er }}$ juillet 2016}

une tendance au dépôt sur le banc sud-ouest et à l'ouest du Grand Port Maritime du Havre entre le banc nord et le Cap de la Hève. La décomposition des flux et bilans sur différentes périodes de l'année a permis de mettre en évidence l'importance du forçage hydrologique (crue/étiage) sur les échanges sédimentaires.

La quantification de la sensibilité des flux sédimentaires face aux conditions hydrométéorologiques permet d'appréhender plus généralement la dynamique sédimentaire des estuaires macrotidaux de types partiellement mélangés. Par la suite, ce travail se poursuivra par l'analyse de ces bilans pour des années aux conditions hydrométéorologiques contrastées (ex. années exceptionnellement sèche/humide, tempétueuse/came).

\section{Remerciements}

Cette étude a été réalisée dans le cadre du projet HYMOSED financé par le programme de recherche Seine-Aval 5 et a bénéficié des mesures in situ collectées dans le cadre du projet SUSPENSE et du réseau de mesure SYNAPSES (GIPSA, page web).

\section{Références bibliographiques}

ARDHUIN F., ROLAND A. (2012). Coastal wave reflection, directional spread, and seismoacoustic noise sources. Journal of Geophysical Research, 117, C00J20, http://dx.doi.org/10.1029/2011JC007832

AVOINE J., ALLEN G.P., NICHOLS M., SALOMON J.C., LARSONNEUR C. (1981). Suspended-sediment transport in the Seine estuary, France: effect of man-made modifications on estuary-shelf sedimentology. Marine Geology, Vol. 40, pp 119-137. http://dx.doi.org/10.1016/0025-3227(81)90046-3

BRENON I., LE HIR P. (1999). Modelling the turbidity maximum in the Seine estuary (France): Identification of formation processes. Estuarine Coastal And Shelf Science, Vol. 49(4), pp 525-544. http://dx.doi.org/10.1006/ecss.1999.0514

GIPSA -Groupement d'Intérêt Public Seine-Aval- (page web). Projet PHRESQUES. URL : http://seine-aval.crihan.fr/web/synapses.jsp?currentNodeId=135

GRASSO F., LE HIR P., BASSOULLET P. (2015). Numerical modelling of mixedsediment consolidation. Ocean Dynamics, Vol. 65(4), pp 607-616. http://dx.doi.org/10.1007/s10236-015-0818-x

GRASSO F., VERNEY R., LE HIR P. (2014). Numerical modelling of the turbidity maximum dynamics in a macrotidal estuary: sensitivity to hydrodynamic and hydrological forcing. PECS 2014 - Physics of Estuaries and Coastal Seas. 17th Edition. 19-23.OCT.2014, Pernambuco State, Brazil, 4 p.

JALÓN-ROJAS I., SCHMID, S., SOTTOLICHIO A., BERTIER C. (2016). Tracking the turbidity maximum zone in the Loire estuary (France) based on a long-term, highresolution and high-frequency monitoring network. Continental Shelf Research, Vol. 117, pp 1-11. http://dx.doi.org/10.1016/i.csr.2016.01.017 
KERVELLA Y., KHOJASTEH POUR FARD I., LE HIR P., RENAULT E., LEMOINE J.-P. (2012). Modélisation hydrodynamique tridimensionnelle en coordonnées curvilignes non-orthogonales de l'estuaire de la Seine. XII ${ }^{\text {èmes }}$ Journées Nationale Génie Côtier - Génie Civil, Cherbourg, France, pp 89-100. http://dx.doi.org/10.5150/jngcgc.2012.010-K

LAZURE P., DUMAS F. (2008). An external-internal mode coupling for a 3D hydrodynamical model for applications at regional scale (MARS). Advances in Water Ressources, Vol. 31, pp 233-250. http://dx.doi.org/10.1016/j.advwatres.2007.06.010

LE HIR P. (2009). Simulation des flux particulaires à l'embouchure de l'estuaire de la Seine: exercice d'estimation des incertitudes. Congrès SHF - 31èmes Journée de l'hydraulique, $9 \mathrm{p}$.

LE HIR P., CAYOCCA F., WAELES B. (2011). Dynamics of sand and mud mixtures: A multiprocess-based modelling strategy. Continental Shelf Research, Vol. 31, pp S135-S149. http://dx.doi.org/10.1016/j.csr.2010.12.009

LE HIR P., FICHT A., JACINTO R.S., LESUEUR P., DUPONT J.P., LAFITE R., BRENON I., THOUVENIN B., CUGIER P. (2001). Fine sediment transport and accumulations at the mouth of the Seine estuary (France). Estuaries, Vol. 24(6B), pp 950-963. http://dx.doi.org/10.2307/1353009

LESOURD S., LESUEUR P., FISSON C., DAUVIN J.C. (In press). Sediment evolution in the mouth of the Seine estuary (France): A long-term monitoring during the last 150 years. Comptes Rendus Geoscience. http://dx.doi.org/10.1016/j.crte.2015.08.001

MARMIN S., DAUVIN J.C., LESUEUR P. (2014). Collaborative approach for the management of harbour-dredged sediment in the Bay of Seine (France). Ocean \& Coastal Management, Vol. 102, pp 328-339. http://dx.doi.org/10.1016/j.ocecoaman.2014.10.012

MCSWEENEY J.M., CHANT R.J., SOMMERFIELD C.K. (2016). Lateral variability of sediment transport in the Delaware Estuary. Journal of Geophysical Research: Oceans, Vol. 121(1), pp 725-744. http://dx.doi.org/10.1002/2015JC010974

TOUBLANC F., BRENON I., COULOMBIER T. (2016). Formation and structure of the turbidity maximum in the macrotidal Charente estuary (France): Influence of fluvial and tidal forcing. Estuarine, Coastal and Shelf Science, Vol. 169, pp 1-14. http://dx.doi.org/10.1016/j.ecss.2015.11.019

VAN LEUSSEN W. (1988). Aggregation of particles, settling velocity of mud flocs a review. In Physical processes in estuaries. Springer Berlin Heidelberg, pp 347-403. http://dx.doi.org/10.1007/978-3-642-73691-9_19

VERNEY R. (2012). Flux de matières en estuaire de Seine. Rapport final du projet FLUMES (Seine-Aval 4), $114 \mathrm{p}$.

YU Q., WANG Y., GAO J., GAO S., FLEMMING B. (2014). Turbidity maximum formation in a well - mixed macrotidal estuary: The role of tidal pumping. Journal of Geophysical Research: Oceans, Vol. 119(11), pp 7705-7724. http://dx.doi.org/10.1002/2014jc010228 